\title{
A comparative study of the efficacy and outcome of methylprednisolone and dexamethasone in moderate to severe COVID-19 disease
}

\author{
Praveen B Gautam ${ }^{1}$, Arunesh Kumar' ${ }^{2}$, Babu Lal Kannoujia ${ }^{3}$, Rajendra Chaudhary ${ }^{4}$ \\ ${ }^{1}$ Assistant Professor and Head, Department of Tuberculosis and Chest Diseases, ${ }^{2}$ Assistant Professor, Department of \\ Community Medicine, ${ }^{3}$ Assistant Professor, ${ }^{4}$ Professor and Head, Department of Medicine, M.V.A.S. Medical College, \\ Basti, Uttar Pradesh, India
}

Background: COVID-19 disease is caused by severe acute respiratory syndrome corona virus 2 (SARS-CoV-2). In the first half of 2020 COVID-19 disease has already converted into a global pandemic. Many treatment options were explored through the world. Aims and Objectives: To compare the efficacy and outcome of methylprednisolone (MTP) and dexamethasone (DEXA) in moderate-to-severe COVID-19 disease. Materials and Methods: In this prospective study, a total of 140 moderate to severe COVID-19 patients were enrolled from 15 April to June 15,2021 . These patients were randomly allocated into two groups, 70 patients were received MTP $2 \mathrm{mg} / \mathrm{kg} /$ day for 3 days followed by $1.0 \mathrm{mg} / \mathrm{kg} / \mathrm{day}$ for 3 days in divided doses while 70 patients received DEXA $8 \mathrm{mg} /$ day in divided dose up to 10 days. Results: The mean age was 45.5 years in MTP group whereas 45.34 years in DEXA group. The clinical outcome in MTP group and DEXA group was assessed in terms of clinical improvement $(92.85 \%$ vs. $81.42 \%)$, radiological improvement $(82.85 \%$ vs. $68.57 \%)$, transfer to ICU $(5.71$ vs. $14.57 \%)$, needs of ventilatory support $(2.85 \%$ vs. $8.57 \%)$ and mortality $(7.14 \%$ vs. $18.57 \%)$ respectively. Conclusion: In this study, MTP demonstrated better outcome as compared to DEXA in COVID-19 patients.

Key words: COVID-19 disease; Dexamethasone; Methylprednisolone

\section{Access this article online}

Website:

http://nepjol.info/index.php/AJMS DOI: 10.3126/ajms.v12i12.39294

E-ISSN: 2091-0576

P-ISSN: 2467-9100

Copyright (c) 2021 Asian Journal of Medical Sciences

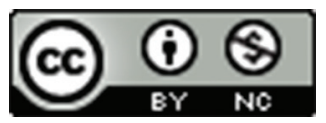

This work is licensed under a Creative Commons Attribution-NonCommercial 4.0 International License.

\section{INTRODUCTION}

Severe acute respiratory syndrome coronavirus-2 (SARS-CoV-2) which causes coronavirus diseases (COVID-19) was first identified in December 2019 in Wuhan city, China, and later spread to many provinces in $\mathrm{China}^{1}$ and has spread with great speed around the world.

On March 12, 2020, it was declared as global pandemic by the World health organization. ${ }^{2}$ The first SARS-CoV-2 positive case in India was reported in the state of Kerala on January $30^{\text {th }}, 2020 .^{3}$
The COVID-19 primarily affects the lung resulting in inflammation and pneumonia. On the basis of clinical, biochemical, and radiological parameters it is divided into mild, moderate, and severe disease. In mild disease, there is fever and upper respiratory symptoms but no documented hypoxia or shortness of breath. In moderate disease, there is tachypnea $>24 / \mathrm{min}$, hypoxia $\left(\mathrm{Spo}_{2}<93 \%\right)$, and in severe disease there is tachypnea $>30 / \mathrm{min}$, hypoxia $(90 \%)$, and biochemical parameter suggest cytokine storm and patient can develop multiorgan failure. ${ }^{4}$

The relatively high infectivity, rapid progression of lung involvement, and absence of definite effective treatment 
all contribute to a need to design effective measures for the management of COVID-19 based on the disease pathogenesis. Although many types of research and studies have contributed to the understanding of the disease and various empirical therapeutic options have been introduced on several operational methods, including the existing and new generation of antivirals and traditional medicine. An effective therapeutic option has not been achieved for severe COVID-19 cases. $^{5-8}$

Corticosteroids have been used previously in respiratory illnesses such as asthma, COPD, severe bacterial pneumonia, and acute respiratory distress syndrome. The use of corticosteroids on COVID-19 disease is still controversial. ${ }^{9}$ Some studies have shown a good response to steroids as they reduce inflammation, ${ }^{10,11}$ However corticosteroids suppress the patients immunity making him more prone to superadded infection. ${ }^{10,12}$ Recently, the recovery trial in the U.K. showed dexamethasone (DEXA) (corticosteroid) to be the only drug able to reduce mortality in severe COVID-19 disease. ${ }^{13}$

However, there is a paucity of literature on the use of methylprednisolone (MTP) in COVID-19. Hence, this study has been under taken to compare the role of DEXA with MTP in patients of COVID-19.

\section{Aims and objectives}

To compare the efficacy and outcome of MTP and DEXA in moderate to severe COVID-19 disease.

\section{MATERIALS AND METHODS}

The current study is a prospective study involving 140 patients diagnosed with moderate to severe COVID-19 disease admitted in Kaily Hospital Mahirshi Vashisth Autonomous State Medical College, Basti, U.P. from 15 April to 15 June 2021.

\section{Inclusion criteria}

- Age: $18-75$ years.

- COVID-19 RT-PCR positive.

- Moderate-to-severe COVID-19 cases according to definition.

- Patients having oxygen saturation $<93 \%$ on room air, regardless of chest X-ray infiltrates.

- Patients given informed consent.

\section{Exclusion criteria}

- Pregnant or lactating females.

- Immunocompromised conditions such as HIV or long-term use of immunosuppressant for any chronic illness.

\section{Study}

We enrolled 140 patients admitted in Kaily hospital Mahirshi Vashisth Autonomous state Medical College, Basti U.P. who fulfilled the inclusion criteria and signed informed consent.

After admission, Baseline oxygen saturation and clinical findings were noted. Chest X-ray was done on admission and laboratory tests were performed such as haemogram, liver function test, kidney function test, C-reactive protein (CRP), Serum ferritin (S. ferritin), lactate dehydrogenase (LDH), and D-dimer. Low molecular weight heparin (Enoxaparin) was prescribed to all patients to prevent thromboembolism and antibiotics also during their hospital stay.

Patients were divided into two groups. Group A ( $\mathrm{n}=70)$ and Group B $(\mathrm{n}=70)$. Group A received intravenous methylprednisolone (MTP) $2.0 \mathrm{mg} / \mathrm{kg}$ bodyweight for 3 days followed by $1.0 \mathrm{mg} / \mathrm{kg}$ for 3 days in the divided dose and Group B received DEXA $8 \mathrm{mg} /$ day intravenously daily in divided dose up to 10 days. Oxygen saturation was recorded daily and laboratory investigations such as CRP, LDH, S. ferritin, and repeat chest X-ray were performed after 5 days and also after 10 days. Patients were given oxygen by nasal cannula, face mask, and non-rebreathing mask. Patients were shifted to ICU if clinical condition deteriorated and/or mechanical ventilation was needed.

We compared outcomes on the basis of clinical, radiological, biochemical parameters and mortality of patients on day 0 that is admission day, $5^{\text {th }}$ and $10^{\text {th }}$ days between both drugs.

Data were analyzed using SPSS 26.0 mean and standard deviation was computed for quantitative variables whereas frequency and percentage were calculated for other category variables.

\section{RESULTS}

A total of 140 patients of COVID-19 having moderate to severe COVID-19 disease and admitted to Kaily Hospital, MVASMC Basti fulfilling inclusion criteria were recruited in the study. Out of 140, 70 patients received MTP (Group-A) and the other 70 patients received DEXA (Group-B).

The mean age in MTP group was found to be 45.5 years and 45.34 years in DEXA group (Table 1).

Both groups were compared for underlying diseases such as diabetes, hypertension, COPD, renal disease, and hypothyroidism. There was no statistically significant 
difference between both groups but hypertension was found in 16 patients in group A while in group B hypertension was found in 26 patients $(\mathrm{P}<0.05)$.

On completion of 10 days of treatment with parental corticosteroids, the biochemical markers of severity improved significantly in MTP as well as DEXA group (Table 2).

Patients were evaluated on day 0,5 , and 10 . There was a significant clinical improvement in the MTP group as compared to the DEXA group $(92.85 \%$ vs. $81.42 \%$, $\mathrm{P}<0.05)$.

Radiological improvement was statistically significant in the MTP group when compared to the DEXA group (82.8\% vs. $68.5 \%, \mathrm{P}<0.05)$.
Transfer to intensive care unit and ventilator requirement was lower in the group that received MTP $(5.71 \%$ vs. $14.28 \%$ ) and $(2.85 \%$ vs. $8.57 \%)$, respectively (Table 3$)$.

Hyperglycemia was more frequent in the DEXA group $(18.57 \%)$ as compared to the MTP group $(7.14 \%)(\mathrm{P}<0.05)$. In terms of mortality, group B reported 13 cases (18.5\%) while in group A five patients died (7.14\%). The difference was statistically significant $(\mathrm{P}<0.05)$.

$\mathrm{Z}$ test for proportion (one-tailed) is carried out to see if there any proportion difference between two drug groups for variables under study. P-value (for one-tailed alternative) showing there is significantly more clinically improved and radiologically improved patients are in MTP group and also for hyperglycemia and mortality, there is significantly lesser proportion of patients in MTP group.

\begin{tabular}{|c|c|c|c|c|c|}
\hline S. No. & Variable & $\begin{array}{l}\text { MTP (Group-A) } \\
n=70\end{array}$ & $\begin{array}{c}\text { DEXA (Group-B) } \\
n=70\end{array}$ & $\begin{array}{c}\text { Total } \\
n=140\end{array}$ & P-value \\
\hline 1. & Age (years) & Mean age 45.50 & Mean age 45.34 & & 0.949 \\
\hline \multirow[t]{3}{*}{2.} & Sex & & & & \\
\hline & Male & 46 (65.71\%) & $50(71.42 \%)$ & $96(68.57 \%)$ & 0.466 \\
\hline & Female & $24(34.28 \%)$ & $20(28.57 \%)$ & $44(31.42 \%)$ & \\
\hline 3. & Diabetes mellitus & $16(22.85 \%)$ & $19(27.14 \%)$ & $35(25.0 \%)$ & 0.612 \\
\hline 4. & CKD & $06(8.57 \%)$ & $07(10.0 \%)$ & $13(9.28 \%)$ & 0.781 \\
\hline 5. & Hypertension & $16(22.85 \%)$ & $26(37.14 \%)$ & $42(30.0 \%)$ & 0.048 \\
\hline 6. & COPD & 09 (12.85\%) & $06(8.57 \%)$ & $15(10.71 \%)$ & 0.438 \\
\hline 7. & Hypothyroidism & $06(8.57 \%)$ & 04 (5.71\%) & $10(7.14 \%)$ & 0.527 \\
\hline 8. & Heart disease & 03 (4.28\%) & 03 (4.28\%) & 06 (4.28\%) & 1.0 \\
\hline 9. & Obesity & $07(10.0 \%)$ & $06(8.57 \%)$ & $13(9.28 \%)$ & 0.781 \\
\hline 10. & Malignancy & $02(2.85 \%)$ & $01(1.42 \%)$ & $03(2.14 \%)$ & 0.563 \\
\hline
\end{tabular}

Table 2: Comparison of intervention between two groups

\begin{tabular}{|c|c|c|c|c|}
\hline Medicine or treatment & Variable & $\begin{array}{c}\text { Mean+SD } \\
\text { MTP } \\
\text { (Group-A) }\end{array}$ & $\begin{array}{c}\text { Mean+SD } \\
\text { DEXA } \\
\text { (Group-B) }\end{array}$ & P-value \\
\hline \multirow[t]{4}{*}{ On day 0} & $\mathrm{SPO}_{2}$ & $89.91 \pm 2.05$ & $90 \pm 1.91$ & 0.799 \\
\hline & $\mathrm{CRP}^{2}$ & $112.49 \pm 46.91$ & $110.62 \pm 48.37$ & 0.816 \\
\hline & S.Ferritin & $186.85 \pm 167.96$ & $190.20 \pm 94.40$ & 0.885 \\
\hline & LDH & $315.63 \pm 184.36$ & $318.40 \pm 131.23$ & 0.9187 \\
\hline \multirow[t]{4}{*}{ Day 5} & $\mathrm{SPO}_{2}$ & $91.90 \pm 1.05$ & $91.00 \pm 2.70$ & 0.013 \\
\hline & $\mathrm{CRP}^{2}$ & $58.62 \pm 39.001$ & $75.52 \pm 50.014$ & 0.032 \\
\hline & S.Ferritin & $134.14 \pm 123.135$ & $142.95 \pm 101.215$ & 0.655 \\
\hline & $\mathrm{LDH}$ & $272.29 \pm 151.83$ & $282.40 \pm 163.16$ & 0.713 \\
\hline \multirow[t]{5}{*}{ Day 10} & $\mathrm{SPO}_{2}$ & $94.48 \pm 81$ & $93.60 \pm 5.411$ & 0.204 \\
\hline & CRP & $28.00 \pm 7.605$ & $31.30 \pm 8.934$ & 0.032 \\
\hline & S. Ferritin & $88.56 \pm 42.82$ & $93.54 \pm 47.14$ & 0.544 \\
\hline & $\mathrm{LDH}$ & $188.49 \pm 47.22$ & $190.28 \pm 39.119$ & 0.823 \\
\hline & & Greenhouse- Geisser $(P=0.00)$ & Greenhouse- Geisser $(P=0.00)$ & \\
\hline
\end{tabular}

CRP: C-reactive protein; S. Ferritin: Serum ferritin; LDH: Lactate Dehydrogenase. Greenhouse-Geisser (P-value) showing there is a significant difference over time within a group for all variables under study. T-test is carried out to see if there any mean difference for variables under consideration for drug $A$ and $B$. At o day, there is no significant difference between drug group $A$ and $B$ for all variables. $(P>0.05)$ At day 5 , $S$. ferritin and LDH showing no difference in drug group $A$ and $B$. While in SPO and $C R P$ there is significant mean difference for the drug. At day 10, only CRP is showing significant mean difference for the two drug group 


\begin{tabular}{|c|c|c|c|c|}
\hline Variables & $\begin{array}{c}\text { MTP } \\
\text { (Group-A) } \\
n=70\end{array}$ & $\begin{array}{c}\text { DEXA } \\
\text { (Group-B) } \\
n=70\end{array}$ & $\begin{array}{c}\text { Total patients } \\
n=140\end{array}$ & P-value \\
\hline Clinically improved & $65(92.85 \%)$ & $57(81.42 \%)$ & $122(87.14 \%)$ & 0.0433 \\
\hline Radiologically improved & $58(82.85 \%)$ & $48(68.57 \%)$ & $106(75.71 \%)$ & 0.0487 \\
\hline Hyperglycemia & $05(7.14 \%)$ & $13(18.57 \%)$ & $18(12.85 \%)$ & 0.046 \\
\hline ICU transfer & $04(5.71 \%)$ & $10(14.28 \%)$ & $14(10.0 \%)$ & 0.090 \\
\hline Ventilator need & $02(2.85 \%)$ & $06(8.57 \%)$ & $08(5.71 \%)$ & 0.145 \\
\hline Hyperglycemic coma/DKA & $0.0(0.0 \%)$ & $0.0(0.0 \%)$ & $0.0(0.0 \%)$ & 0 \\
\hline Superadded infection & $0.0(0.0 \%)$ & $0.0(0.0 \%)$ & $0.0(0.0 \%)$ & 0 \\
\hline Mortality & $05(7.14 \%)$ & $13(18.57 \%)$ & $18(12.85 \%)$ & 0.0433 \\
\hline
\end{tabular}

CKD: Chronic kidney disease, MTP: Methylprednisolone, DEXA: Dexamethasone

For other variables, the result is coming not out to be significant.

\section{DISCUSSION}

COVID-19 disease is caused by SARS-CoV-2 has emerged as a major threat globally. The novelty of disease and its undesirably high morbidity and mortality have lead physicians to explore new dimensions of treatment.

ARDS and cytokine release syndrome are dreadful complications of COVID-19, both characterized by increased levels of Tumor necrosis factor Alfa, Interleukin (IL) IB, IL-2, IL-6, IL-8, IL-10, and Interferon-gamma which causes dysregulated auto-inflammatory response, severe tissue inflammation and ultimately death. ${ }^{14}$ Due to its anti-inflammatory properties corticosteroids have been used in the treatment of COVID-19. We performed a comprehensive comparison of MTP and DEXA in the treatment of moderate to severe COVID-19 disease.

In the current study, we included 140 patients with moderate to severe COVID-19 disease, they were randomly allocated to two groups, i.e., Group A (MTP group) and group B (DEXA group). Both groups were matched regarding their demographic profile (Table 1).

In MTP group, a more significant decrease in inflammatory response was observed than in DEXA group leading to decrease in CRP, LDH, and S. ferritin. This is consistent to the finding of Pinzón et al. ${ }^{15}$

In a randomized clinical trial performed by Edalatifard et al., the efficacy of intravenous MTP pulse was compared with standard care. In the mentioned study, patients with clinical improvement were higher in MTP group than in the standard care group $(94.1 \%$ vs. $57.1 \%)$. The clinical improvement was noted in terms of increase in oxygen saturation and alleviation of myalgia, chest pain, cough, fever, and gastrointestinal symptoms. ${ }^{16}$ These findings were in line with our study. The present study showed a significant clinical improvement ( $92.8 \%$ vs. $81.4 \%$ ) as well radiological improvement on ( $82.8 \%$ vs. $68.5 \%)$ in MTP group when compared with DEXA group (Table 2).

As per study performed by Pinzon et al., patients in DEXA group developed severe ARDS in higher proportion than the MTP group, and transfer to the intensive care unit was less in the MTP group which is related to less clinical deterioration and less progress to critical illness with the administration of high dose of MTP, ${ }^{15}$ this was in corroboration with the finding of our study where transfer to ICU was $5.7 \%$ in MTP group as compared to $14.2 \%$ in DEXA group.

In a retrospective cohort study conducted by Wang et al., in the evaluation of treatment of patients suffering from COVID-19 with low dose of MTP (1-2 mg/ $\mathrm{kg} /$ day) for 5-7 days had shorter hospital stay and less requirement of mechanical ventilation. ${ }^{10}$ These findings were in accordance to the current study $(2.8 \%$ in MTP group vs. $8.5 \%$ in DEXA group).

Treatment of COVID-19 patients with corticosteroids may have some complications like superimposed infection, immunosuppression, and hyperglycemia. Hyperglycemia was more frequent in DEXA group $(18.57 \%)$ when compared to MTP (7.14\%). The hyperglycemia was managed effectively with insulin and none of the patients developed serious complications such as ketoacidosis or hyperosmolar coma. In some studies, it was found that hyperglycemia was more frequent in those who received MTP, managed without substantial complication. . $^{10,16,17}$

None of our patients developed superadded bacterial pneumonia as determined by pro-calcitonin levels and serial chest X-ray for new opacities. This could be explained by short period of corticosteroid use (i.e. 5 days). This is similar to the study performed by Fatima et al. ${ }^{18}$ 
The recovery trial showed significantly less mortality in DEXA group as compared to standard care group..$^{13}$ However, the recovery trial did not study the effects of MTP.

Wang et al., conducted a retrospective cohort study of MTP therapy in severe patients of COVID-19 pneumonia and found that there was a significant reduction in morbidity and mortality with MTP. ${ }^{10}$

$\mathrm{KO}$ et al., concluded that therapeutic benefits of corticosteroids are not limited to DEXA only. The higher anti-inflammatory potency of MTP had greater mortality benefits in COVID-19 patients requiring mechanical ventilation. ${ }^{19}$

In the present study, there was a significant reduction of mortality in the MTP group as compared to DEXA group (7.14\% vs. $18.5 \% ; \mathrm{P}<0.05)$. This is similar to the Edalatifard et al., who showed lower mortality rate in MTP group $(5.9 \%$ vs. $42.9 \%, \mathrm{P}<0.0001) .{ }^{16}$

\section{Limitations of the study}

To the best of our knowledge, this was the first study in eastern Uttar Pradesh, India comparing the efficacy of MTP with DEXA.

However, major limitations of this study are sample size is small and there was no follow-up of patients after discharge.

\section{CONCLUSION}

In this study, the severity markers of COVID-19 pneumonia such as CRP, LDH, and S. ferritin are significantly reduced by administration of both MTP as well as DEXA. However, the outcome in the terms of clinical improvement, radiological improvement, reduction in mechanical ventilation requirement, and decrease in mortality was found to be better in MTP group when compared with DEXA group.

\section{ACKNOWLEDGMENT}

We are thankful to Mr. Pawan Kumar biostatistician for helping in statistical analysis.

\section{REFERENCES}

1. World Health Organization. Situation Report-109. Coronavirus Disease 2019 (COVID-19). Geneva: World Health Organization; 2020. Available from: https://www.who.int/emergencies/ diseases/novel-coronavirus-2019/situation-reports [Last accessed on 2020 May 09].
2. World Health Organization. WHO Announces COVID-19 Outbreak a Pandemic. Geneva: World Health Organization; $2020 . \quad$ http://www.euro.who.int/en/health-topics/healthemergencies/coronavirus-covid-19/news/news/2020/3/whoannounces-covid-19-outbreak-a-pandemic [Last accessed on 2020 Mar 12].

3. Indian Council of Medical Research. COVID-19 ICMR. COVID-19. Indian Council of Medical Research. Government of India. Indian Council of Medical Research; 2020. Available from: https://main.icmr.nic.in/content/covid-19 [Last accessed on 2020 May 09].

4. Ministry of Health and Family Welfare Government of India. AlIMS/ ICMR-COVID-19 National Task Force/Joint Monitoring Group (Dte.GHS) Ministry of Health and Family Welfare Government of India. Clinical Guidance for Management of Adult COVID-19 Patients. Department of Medicine AlIMS ND, April. New Delhi: Ministry of Health and Family Welfare Government of India; 2021.

5. Cheng ZJ and Shan J. 2019 novel coronavirus: Where we are and what we know published correction appears in infection. Infection 2020;48(2):155-163. https://doi.org/10.1007/s15010020-01401-y

6. Young B, Tan TT and Leo YS. The place for remdesivir in COVID-19 treatment. Lancet Infect Dis. 2021;21(1):20-21. https://doi.org/10.1016/S1473-3099(20)30911-7

7. Vetter $P$, Kaiser L, Calmy A, Agoritsas $T$ and Huttner A. Dexamethasone and remdesivir: Finding method in the COVID-19 madness. Lancet Microbe. 2020;1(8):e309-e310. https://doi.org/10.1016/S2666-5247(20)30173-7

8. Shahriarirad R, Khodamoradi Z, Erfani A, Hosseinpour $H$, Ranjbar K, Emami Y, et al. Epidemiological and clinical features of 2019 novel coronavirus diseases (COVID-19) in the South of Iran. BMC Infect Dis. 2020;20(1):427. https://doi.org/10.1186/ s12879-020-05128-x

9. Cascella M, Rajnik M, Cuomo A, Dulebohn SC and Di Napoli R. Features, Evaluation, and Treatment of Coronavirus (COVID-19). Treasure Island, FL, StatPearls Publishing; 2020.

10. Wang $Y$, Jiang $W, \mathrm{He} Q$, Wang $C$, Wang $B$, Zhou $P$, et al. A retrospective cohort study of methylprednisolone therapy in severe patients with COVID- 19 pneumonia. Signal Transduct Target Ther. 2020;5(1):57. https://doi.org/10.1038/s41392020-0158-2

11. Ye Z, Wang $Y$, Colunga-Lozano LE, Prasad M, Tangamornsuksan W, Rochwerg B, et al. Efficacy and safety of corticosteroids in COVID-19 based on evidence for COVID-19, other coronavirus infections, influenza, community-acquired pneumonia and acute respiratory distress syndrome: A systematic review and meta-analysis. CMAJ. 2020;192(27):E756-E767. https://doi.org/10.1503/cmaj.200645

12. Zhang W, Zhao Y, Zhang F, Wang Q, Li T, Liu Z, et al. The use of anti-inflammatory drugs in the treatment of people with severe coronavirus disease 2019 (COVID-19): The Perspectives of clinical immunologists from China. Clin Immunol. 2020;214:108393. https://doi.org/10.1016/j.clim.2020.108393

13. Wilkinson E. RECOVERY trial: The UK covid-19 study resetting expectations for clinical trials. BMJ. 2020;369:m1626. https:// doi.org/10.1136/bmj.m1626

14. Ragab D, Eldin HS, Taeimah M, Khattab R and Salem R. The COVID-19 cytokine storm; what we know so far. Front Immunol. 2020;11:1446. https://doi.org/10.3389/fimmu.2020.01446

15. Pinzón MA, Ortiz S, Holguín $H$, Betancur JF, Arango DC, Laniado $\mathrm{H}$, et al. Dexamethasone vs methylprednisolone high dose for Covid-19 pneumonia. PLoS One. 2021;16(5):e0252057. https://doi.org/10.1371/journal

16. Edalatifard M, Akhtari M, Salehi M, Naderi Z, Jamshidi A, Mostafaei $\mathrm{S}$, et al. Intravenous methylprednisolone pulse as a treatment for hospitalized severe COVID-19 patients: Results from a randomised 
controlled clinical trial. Eur Respir J. 2020;56(6):2002808. https:// doi.org/10.1183/13993003.02808-2020

17. Corral L, Bahamonde A, de las Revillas FA, Gomez-Barquero J, Abadia-Otero J, Garcia-lbarbia C, et al. GLUCOCOVID: A controlled trial of methylprednisolone in adults hospitalized with COVID-19 pneumonia. MedRxiv. 2020;2020:20133579. https://doi.org/10.1101/2020.06.17.20133579

18. Fatima SA, AsifM, Khan KA, Siddique N and KhanAZ. Comparison of efficacy of dexamethasone and methylprednisolone in moderate to severe covid 19 disease. Ann Med Surg. 2020;60:413-416. https://doi.org/10.1016/j.amsu.2020.11.027

19. Ko JJ, Wu C, Mehta N, Wald-Dickler N, Yang W and Qiao R. A Comparison of Methylprednisolone and dexamethasone in intensive care patients with COVID-19. J Intensive Care Med. 2021;36(6):673-680. http://doi. org/10.1177/0885066621994057

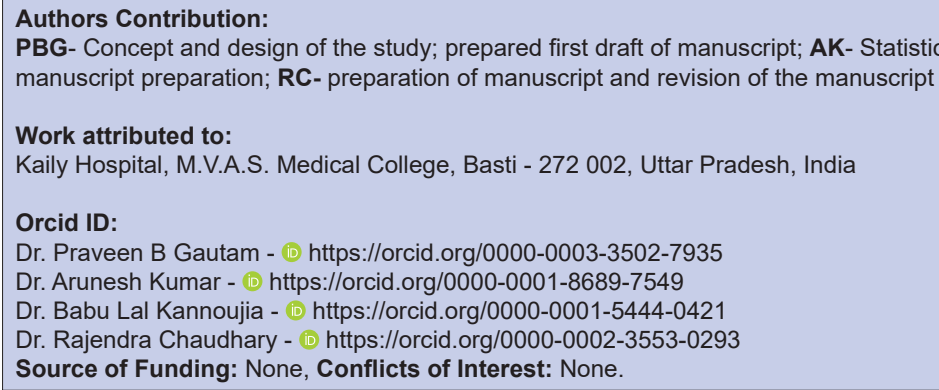

